\title{
The association of adherence to moringa, plantains, moringa-plantain combined diets, and other plant-based dietary patterns with glycemic control among persons with T2DM, a hospital based cross sectional survey in Ghana
}

Be-Ikuu Dominic Doglikuu ${ }^{1,3}$, Abdulai Abubakari ${ }^{4}$, Mehdi Yaseri ${ }^{5}$, Elham Shakibazadeh ${ }^{6}$, Abolghassem $^{2}$ Djazayery $^{2 *}$ Khadijeh Mirzaei $^{2 *}$

${ }^{1}$ International Campus, Department of Community Nutrition, School of Nutritional Sciences and Dietetics,

Tehran University of Medical Sciences (TUMS), Tehran, Iran

${ }^{2}$ Department of Community Nutrition, School of Nutritional Sciences and Dietetics, Tehran University of Medical Sciences (TUMS), Tehran, Iran.

${ }^{3}$ Ministry of Health, Nursing and Midwifery Training College, TwifoPraso, Central Region, Ghana.

${ }^{4}$ Department of Global and International Health, School of Public Health, University of Development Studies (UDS), Tamale, Ghana.

${ }^{5}$ Department of Epidemiology and Biostatistics, School of Public Health, Tehran University of Medical Sciences, Tehran, Iran

${ }^{6}$ Department of Health Education and Promotion,School of Public Health, Tehran University of Medical

Sciences, Tehran, Iran

*Corresponding Authors

Emails:mirzaei_kh@sina.tums.ac.ir

Email: djazayery@yahoo.com/ jazaiers@tums.ac.ir 


\begin{abstract}
Diabetes mellitus (DM) is an independent risk-factor for cardiovascular diseases. Plant-based dietarypatterns have been shown to positively impact the effects this cardiovascular risk-factor. The purpose of this study is to investigate the association of adherence to moringa, plantains, moringa-plantain combined, and other plant based dietary-patterns with glycemic-control among persons with type-2diabetes Mellitus (T2DM). Facility-based cross-sectional-study was conducted among 530 T2DM patients in Ghana. Structured-questionnaires were used to collect demographic, anthropometric, and clinical variables. Adherence to plant-based dietary-patterns were assessed with 24-hour dietaryrecall questionnaire. SPSS version-22 was used in data analysis. BMI, HbA1c\%, HDL-cholesterol and LDL-cholesterol were significantly correlated with adherence to plant-based dietary-patterns (pvalue $<0.05)$. After adjusting for confounders, adherence to: Plantain diets, standardized regression coefficient $\beta$ (95\%CI): -0.098 (-0.321, -0.022), Yam, $\beta$ (95\%CI): $0.148(0.066,0.496)$, Moringa diets $\beta$ (95\%CI): -0.095 (-0.325,-0.011) and Bean-diets $\beta$ (95\%CI): -0.112 (-0.577-.007) were significantly associated with glycemic control. Also adherence to: Plantain-moriga combined diets $\beta(95 \% \mathrm{CI})$ : 0.406 (-0.413, -0.049) and Plantain-beans combined diets $\beta$ (95\%CI): $-0.128(-0.188,-0.038)$ were significantly associated with glycemic control. Adherence to Plantain, Yam, Beans, Plantain-moriga combined diets, and Plantain-beans combined diets could be associated with glycemic control.
\end{abstract}

Keywords: Diabetes mellitus, Adherence, Moringa, Plantain, Glycemic control, Ghana.

\title{
Introduction
}

Diabetes mellitus (DM) is a global public health problem and an independent risk factor for cardiovascular disease[1]. People with diabetes have 2-4 times increased risk for heart diseases or stroke compared with those without diabetes[2]. Studies among Seventh-day Adventists congregants reported that the prevalence of type 2 diabetes(T2DM) among people consuming vegetarian diets was approximately half of those following omnivorous diets [3, 4]. Other cohort studies also suggested that meat consumption is associated with increased risk of type 2 diabetes[5,6]. In clinical trial, glycemic control was found to improve among persons who switched from consuming omnivorous diets to vegetarian diets[7], suggesting that vegetarian or plant based dietary factors may be beneficial in preventing and treating T2DM [8,9]. Although plant-based dietary patterns have been shown to play significant roles in DM and body weight management, the question "'which plant-based products 
have the greatest benefits for glycemic controls, cardiovascular risk factors, and weight management" continue to remain controversial. This controversy arise because many studies exploring the association of vegetarian or plant-based dietary patterns with diseases prevention or control usually have relatively short durations (e.g., <4 weeks), small sample sizes, and some also failed to control the effects of some confounders including physical activity on these dietary patterns for disease prognoses. These therefore limit the ability of nutrition researchers to strongly draw conclusions on the effectiveness of plant-based dietary patterns on disease prevention and control.

Despite this bottleneck however, studies show that individuals who follow plant-based dietary patterns typically consume fewer calories, less: saturated fat, cholesterol; and body mass indexes (BMIs) than non-vegetarians [10-12]. These individuals also tend to consume more fiber, potassium, and vitamin $\mathrm{C}$. In prospective cohort studies among adults vegetarians, compared non-vegetarians, it was found that vegetarians eating patterns were associated with lower prevalence of type 2 diabetes[7] cardiovascular disease (CVD)[13] hypertension[14], obesity [15, 16] and reduced usage of medical care [17]. Recently, the uniform calorie-controlled diet plans for parsons with diabetes have been replaced with individualized meal-planning approaches. In line with this, nutrition guidelines have now tend to focus more on carbohydrate counting and minimizing saturated and trans fats intakes. This has therefore prompted both the American Academy of Nutrition and Dietetics (AAND) and the American Diabetes Association (ADA) to called for the inclusion of well-planned, plant-based (vegetarians and vegans) dietary patterns in meal-planning options for people with diabetes[18, 19]. Despite this land mark recommendation and paradigm shift, enough studies have not been carried out by nutrition scientist to support evidence and identify specific plant-based products that would have the greatest benefits for disease prevention and health promotion. In this regard, our study therefore seeks to assess the association of adherence to moringa, plantain, moringa-plantain combined diets, and other plant based dietary patterns with glycemic control among persons with T2DM in Ghana

\section{Method}

Hospital based cross-sectional survey was conducted among 530 individuals living with DM in Brong Ahafo Region (BAR), Ghana. Single population proportion formula ( $n=\frac{\mathrm{Z}^{2} \mathrm{P}(1-\mathrm{P})}{e^{2}}$ ) was used to determine the sample size. The letter ' $n$ ' in the formula for the sample size determination denotes the study sample size, ' $\mathrm{Z}$ ' is normal distribution of 1.96 for $95 \%$ confidence interval, ' $\mathrm{P}$ ' is the true 
population proportion of adherence to dietary recommendation among persons with DM in the study area (Brong Ahafo region) and 'e' is standard error (5\%). Previous study in Brong Ahafo region of Ghana, reports that prevalence of adherence to dietary recommendation is $68.5 \%$ [20]. Substituting these values in the equation above, the sample size ' $\mathrm{n}$ ' was calculated as $n=$ $\frac{1.96^{2} * 0.685(1-0.685)}{0.05^{2}}=332$. However, in the event of non-response and registration error, a contingency sample of $60 \%$ was considered in the sampling. Therefore, the final sample size was $0.6 * 332=531.2 \approx 532$.

\section{Sampling}

Individuals 18 years and above who were diagnosed with T2DM by physicians, using the ADA diagnostic and classification guideline 2011[21], and counseled to follow recommended dietary guidelines for at least 3 months and over were sampled into the study. Participants' 70 years and above who could not answer interview questions, mentally incapacitated, and severely ill were excluded from the studies. Likewise pregnant and lactating mothers were excluded. Simple random sampling was used to select the 6 hospitals, and the eligible participants in these hospitals were consecutively enrolled through systematic random sampling.

\section{Ethical Approval}

The study protocol was approved by Ghana Health Service Ethics Review Committee (GHSERC008/08/18) and Tehran University of Medical Sciences Ethics Review board (IR.TUMS.VCR.REC.1397.409). All participants were requested to sign an informed consent form before participating in the study. This study was performed in accordance with the Declaration of Helsinki for humans and laboratory animal research.

\section{Assessing Demographic, Anthropometric and Clinical Characteristics}

Age, diabetes-duration, medications intakes and other demographic characteristics of participants were assessed using structured questionnaires while weight and height were measured using adult 
weighing scale and stadiometer. The reading obtained were recorded to the nearest $0.5 \mathrm{~kg}$ and $0.5 \mathrm{~m}$ respectively. These measurements were taken when participants were asked to be in light clothes without shoes, and were in standing position. Body mass index (BMI, $\mathrm{kg} / \mathrm{m} 2$ ) was calculated by dividing weight in kilograms with square meters height. Systolic and diastolic blood pressures were measured using manual sphygmomanometer and stethoscope. The reading obtained were also recorded to the nearest $0.5 \mathrm{mmHg}$ after participants were allowed to relax for 5 or more minutes.

\section{Assessing Adherence to Plant-based (Moringa, Plantain, Moringa-Plantain Combined, and other Plant-based) Dietary Patterns}

Participants' dietary intakes were assessed using 24-hour dietary recall questionnaire. Participants' were asked to respond to a three separate 24-hour dietary recall questionnaires (one on Monday, Wednesday, and Saturday). This was done to evaluate participants' true dietary intakes in a typical day. They were asked to report details intakes of all foods and drinks/beverages in each previous day prior to the interview. They were also asked to report in detail the foods preparation method, the portion size they were served and the actual amount they ate. The information obtained were summed up and analyzed using Ntri.IV software.

\section{Assessing Physical Activity Levels}

World Health Organization (WHO) Physical Activity Questionnaires were used to assess participants' physical activity level. We also used the WHO recommended guideline in analyzing the physical activity reports obtained from participants. In calculating participants' physical activity level, WHO recommends the total time spent in doing the physical activity including activity: for work, during transport and in leisure time, and the intensity of the physical activity in a typical week should be observed. WHO physical activity guideline also recommends that adults should do at least 150 minutes of moderate-intensity physical activity OR 75 minutes of vigorous-intensity physical activity OR an equivalent combination of moderate- and vigorous-intensity physical activity achieving at least 600 MET-minutes for health. For work related physical activity, individuals who 
reported moderate work were scored MET value =4.0; those who reported vigorous work were scored MET value $=8.0$. For transport related physical activity, participants who reported cycling and walking were given MET value $=4.0$. Those who engaged in recreational physical activities were given MET value $=4.0$ for moderate recreational activities and $\mathrm{MET}$ value $=8.0$ for vigorous recreational physical activities. The MET values of each physical activity level was multiply by the number of hours and days spent in doing that particular physical activity. The results obtained were grouped with reference to the WHO physical activity guideline cut off points ("Not meet recommendations for Total Physical Activity" MET minutes per week < 600) and ("Meet recommendation for Total Physical Activity", MET minutes per week are $\geq 600$ )[22]

\section{Laboratory/Biochemistry Analysis}

Whole blood sample was used to estimates HbA1c by turbidimetric inhibition immunoassay method using Cobas Integra automated Chemistry analyzer (Roche Cobas Integra 400 Plus, Roche Diagnostics, USA) [23]. Participants' overnight fasting blood samples were collected in EDTA test tubes to prevent cross reaction prior to analysis. The well-mixed EDTA-anti-coagulated whole blood was later transferred into sample test-tubes and placed on a rack. The red blood cells in the test tubes were hemolyzed using low osmotic pressure and the free hemoglobin degraded with pepsin to liberate the $\mathrm{N}$-terminal of the beta chain ( $\beta$-N-terminal) of the HbAlc. The HbA1c $\beta$-N-terminal was then bind with latex particles-bound monoclonal antibodies while the remaining free antibodies were agglutinated using synthetic polymers. This process formed multiple copies of $\beta$-N-terminal structure of $\mathrm{HbA1c}$, and therefore leave the test sample turbid in the test tube. The change in turbidity of the sample was then measured at $552 \mathrm{~nm}$ and the final $\mathrm{HbA1c}$ values were expressed as a percentage using the formula: HbA1c $(\%)=(\underline{\mathrm{HbA} 1 \mathrm{c}}) \times 100$

\section{$\mathrm{Hb}$}

The test was standardized with an intra-assay coefficient of variation (CVs) 0.9-1.5\% and inter-assay CVs 1.1-1.6\%. Daily calibrations and maintenance of the analyzer were performed according to the manufacturer's instructions[24]. Quality control was maintained using the quality control procedures provided with the analyzer by the manufacturer [negative and positive controls (high and low HbA1c)]. Other biochemical parameters such as low-density lipoprotein (LDL), high-density lipoprotein (HDL), triglycerides (TG), total cholesterol (TC), serum bicarbonate, serum creatinine 
(Cr) blood urea nitrogen (BUN) etc. were obtained from participants' past two to three months medical records in the hospital.

\section{Statistical Analysis}

IBM SPSS version 22.0 (SPSS, Chicago, IL, USA) was used in data analysis. Data normal distributions were checked with Kolmogorov-Smirnov test. Descriptive statistics were used to describe participants' demographic characteristics, while Pearson correlation tests were used to test correlation between demographic, anthropometry and clinical variables with adherence to plant-based dietary patterns. Finally multiple linear regression models were used to assess the association of adherence to plant-based dietary patterns and glycemic control. Multiple linear regression model looks at the association between predictor variables on one dependent variable in an equation $\mathrm{Y}=$ $a+b_{1} X_{1}+b_{2} X_{2}+{ }_{b 3} X_{3}+b_{4} X_{4}+b_{5} X_{5}+b_{k} X_{k}+e$, where ' $a$ ' is the regression constant, $b$ is the regression coefficient, $\mathrm{X}_{1} \ldots \ldots \mathrm{X}_{\mathrm{k}}$ are the independent variables and ' $\mathrm{e}$ ' is the variance of the population mean distributions. This statistic was used in our study because our dependent variable (Adherence to plant-based dietary pattern) is normally distributed and has equal variance around the mean. The independent variables also have linear relationships with no multicollinearity. Furthermore, our sample size is fairly large and is said to have fair representation of the larger population which met the assumption for the statistics used. We set all variables statistical significant at 0.050 alpha levels in our analysis.

\section{Results}

Participants' demographic characteristics and clinical variables were presented in Table 1. Mean and standard deviation of age, duration lived with DM and adherence to plant-based dietary patterns were 58.10(9.70), 4.90(5.39) and 32.56(9.61) respectively. Majority (70.9\%) of the participants were female, have: no education (38.1\%), junior high (24.9\%) and university education (3.0\%). Mean and standard deviation of systolic and diastolic blood pressure were 135.67(20.44) and 77.79 (12.79) 
respectively. Mean and standard deviation of HbA1c\%, Triglyceride, HDL-cholesterol and LDLcholesterol were 8.12(3.34), 4.63(14.14), 1.73(0.90) and 5.15(3.40) respectively.

Table 2 presented the correlation of participants' demographic characteristics and clinical variables with adherence to plant-based dietary pattern. Significant correlation was found between BMI, HbA1c\%, HDL-cholesterol and LDL-cholesterol with adherence to plant-based dietary pattern (pvalue $<0.05)$.

The association of moriga, plantain, moringa-plantain combined diets and other plant-based dietary patterns with glycemic control were presented in Table 3. Statistically significant associations were found for adherence to plantain diets, Standardized regression coefficient $\beta$ (95\%CI): -0.098 (-0.321, -0.022), yam diets $\beta$ (95\%CI): 0.148 (0.066, 0.496), moringa leaves diets $\beta$ (95\%CI): -0.095 (-0.325,$0.011)$ and bean diets $\beta(95 \% \mathrm{CI})$ : -0.112 (-0.577-.007) with glycemic control. Furthermore, significant associations were found for plantain-moriga combined diet $\beta(95 \% \mathrm{CI})$ : $-0.406(-0.413,-0.049)$ and plantain-beans stew combined diet $\beta(95 \% \mathrm{CI})$ : $-0.128(-0.188,-0.038)$ with glycemic control

\section{Discussions}

Many studies have touted the benefits of plant-based dietary patterns for health promotion and disease prevention [25-27]. However most of these studies tend to have low sample sizes, relatively short follow up periods and failed to adjust the effect of physical activities on these dietary patterns. The first objective of our study was to establish whether significant correlations exist for participants' demographic characteristics, anthropometric and clinical variables with adherence to plant-based dietary patterns among persons with T2DM. Secondly we sought to establish whether significant associations exist between adherences to plant-based (Moringa, Plantain, Moringa-Plantains combined) dietary patterns with glycemic control among persons with T2DM, after we have adjusted the effect of physical activity on plant-based dietary intakes. At the end of the study we found that significant correlations exist between BMI $\left(\mathrm{Kg} / \mathrm{m}^{2}\right)$, $\mathrm{HbA} 1 \mathrm{c} \%$, Total-cholesterol (mmol/L), HDLcholesterol (mmol/L) and LDL-cholesterol (mmol/L) with adherence to plant-based dietary patterns. We also found significant associations between adherences to plantain, yam, moringa, Beans, plantain-moriga combined diets, and plantain-beans combined diets with glycemic control among persons with T2DM. 
Epidemiological studies[28, 29] have touted the benefits of plant-based dietary patterns for health promotion and disease prevention. These evidences together with other empirical observations have led both the AAND and the ADA to make landmark recommendation for the inclusion of wellplanned, plant-based dietary patterns in meal-planning for persons with diabetes. Despite these remarkable recommendations, the supremacies of some plant-based dietary patterns over others for disease prevention (glycemic control) have not been clearly established. Our study therefore seeks to highlight on this.

In our study we noticed that adherence to plantain diets were significant with glycemic control (decreased HbA1c \%) among persons with T2DM. This finding could be true because plantain (Musa paradisiaca) is known to have low glycemic index (GI) with high fiber and resistant starch content[30]. Globally, plantain is a staple crop in the humid and sub-humid parts of Africa, Asia, Central and South America that is usually eaten as an energy yielding food by many. In some folktale medicine, plantain has been used in the management of diabetes, renal and liver dysfunction[31]. These foods have been found to be higher in fiber and resistant starch [32]. These nutritional properties make plantains aid in digestion and add bulkiness to foods in the gastrointestinal tract. The bulkiness effect of plantain diets make individuals who consumed them feel full upon fewer consumption, and with fewer calories intake which are essential in weight management and the prevention of the negative effects of hyperglycemia in DM patients. Diabetic ketoacidosis (DKA) and hyperosmolar hyperglycemic syndrome (HHS) are the two acute and life threatening complications arising from DM that require immediate medical attention[33]. These conditions occurred as a result of improperly controls of blood glucose. Patients with DKA present with hyperglycemia, ketonemia, and metabolic acidosis, while those with HHS present with extreme hyperglycemia, increased osmolality, and severe dehydration. These acute complications can be life threatening if left untreated. Since plantain diets have been shown to have positive effects in glycemic control among patients with DM, it can be recommended to them. Although we have not found any clinical trial on human beings using plantain diets in diabetes management, studies among laboratory animals showed significant improvement in glycaemia and other metabolic parameters when these animals were fed on plantain diets and compared with others fed on different foods [34-36]. These finding therefore highlighted the important and critical roles plantain diets can play in DM management. In line with this it is important that healthcare workers, identify the complementary 
roles plantain diets have in DM management and confidently recommend them to persons with living with DM disease management.

In this study we also noticed that participants who adhere to moringa diets, and beans diets were shown to have significant improved glycemic control (decreased HbA1c \%). These findings too could be true because beans and moringa (Moringa oleifera) have been shown to have low glycemic indexes [37]. Moringa oleifera also called the drumstick tree, miracle tree, ben oil tree, or the horseradish tree, is found in Africa, India, Asia, and South America [38]. It has been used for centuries due to its medicinal properties and health benefits. Recently it is known that moringa contains variety of proteins, vitamins, minerals, and other functions such as antifungal, antiviral, antidepressant, and anti-inflammatory properties [39]. Moringa plants are widely found in Ghana, growing from north to south in backyard gardens as vegetables or on the field as wild weeds and herbs. Although it is not the standard practice of nutrition scientists and dietetic professionals in Ghana to put patients on moriga diets for health promotion and disease treatments purposes, patients who voluntary consumed these plants and their products as foods, were shown to have better health outcomes when compared with others. Moringa is a multipurpose plant consumed as food and known for its medicinal uses, among others. The leaves, seeds and pods of moringa are the main parts usually used as foods or food supplements. These products are nutritionally rich, and have high polyphenol content in the form of phenolic acids, flavonoids and glucosinolates, and are shown to exert numerous in vitro and in vivo effects, including hypoglycemic activity[40]. Although different hypoglycemic drugs are used in DM management, there is still a growing interest in the use of these medicinal plants due to their low price, easy availability, and fewer or no side effects. In our study we found that moringa-plantain combined diets and plantain-beans combined diets were significantly associated with improved glycemic control (decreased HbA1c \%). These findings too were interesting. Because in the univariate analyses, we realized that plantain, moringa, and beans diets had independently improved glycemic control (decreased HbA1c \%). We therefore expected that combining these products in one diet could improve glycemia significantly, which has been confirmed. Studies in different countries have highlighted moringa, plantains and beans as important and miracle foods in glycemic control. However, with the effect of confounding variables such as exercise, alcohol intakes, and smoking on these diets, the acceptability of the protective effects of these plant diets in DM management has become challenging. Although our study sought to address some of these challenges, some limitations 
with our study also make it difficult for us to draw definite conclusions based on our findings. Some of these limitations are: Our study has relatively small sample size (530 participants) with very low power to detect true associations. We also adopted a relatively weak study method (cross sectional study) to investigate this problem. Since cross sectional study cannot detects causal associations, we are therefore constraint to say that significant causality exist between moringa, plantain, moringaplantain combined diets and other plant-based dietary patterns with glycemic control. Despite these limitations, our study however demonstrates that controlling the effect of physical activity; moringa, plantain, moringa-plantain combined diets and other plant-based dietary patterns could stand out as candidates in improving glycemia among persons with T2DM.

\section{Conclusion}

Diabetes mellitus is an independent risk factor for cardiovascular diseases and stroke. People with diabetes have 2-4 times increased risk for heart disease or stroke. Dietary recommendations with emphasis on plant-based dietary patterns have been highly touted to reverse these cardiovascular risk factors among persons with DM. However the supremacies of some plant-based diets over others have not been established. Also the confounding effect of some behaviors such as exercise, alcohol consumption and others variables among persons with DM make it difficult to draw strong conclusion on the causal association of plant-based dietary patterns with diseases prevention and health promotion. In our study we found that adjusting for the effect of exercise on plant-based dietary intake plantain, yam, bean, plantain-moriga combined diets and plantain-beans combined diets were significantly associated with glycemic control among persons with T2DM. These evidences therefore suggest that health care workers should prioritized plant-based dietary patterns with more focus on plantains, moringa, beans, plantain-moringa combined diets and plantain-beans combined diets in diseases prevention and health promotion purposes.

\section{Acknowledgment}

We acknowledge the regional director of health services in Brong Ahafo region, all the medical superintendents, and laboratory scientists in the various hospitals who provided technical support in this study. We also acknowledge all the participants who took part in this study.

\section{Authors' contribution}


BDD collected and analyzed the data and wrote the manuscript.

AA obtained ethical clearance in Ghana for this study, supervised the data collection, and proofread the final version of the manuscript before submission.

YM supervised the data analysis and proofread the final version before submission.

SE proofread the final version of the manuscript before submission.

$\mathrm{AD}$ acquired funding for the study, supervised the data collection process, and proofread the final version of the manuscript before submission.

MK supervised, coordinated the study, took part in the data collection took $\mathrm{p}$ art in the data analysis and wrote the manuscript.

\section{Competing interests}

All authors have read and approved the manuscript, and declared no conflict of interest.

\section{Funding Source}

This study was funded by Tehran University of Medical Sciences.

\section{Availability of data and material}

The datasets generated and/or analyzed during the current study are not publicly available but are available from the corresponding author on reasonable request.

Table 1: Participants demographic and clinical variables

\begin{tabular}{|c|c|c|}
\hline Demographic Variables & $\mathbf{N}(\%)$ & Means(SD) \\
\hline Age (years) & & $58.10(9.70)$ \\
\hline Duration lived with Diabetes (years) & & $4.90(5.39)$ \\
\hline $\mathrm{BMI}\left(\mathrm{Kg} / \mathrm{m}^{2}\right)$ & & $23.14(2.92)$ \\
\hline Number of cigarette smoke per day & & $0.16(0.49)$ \\
\hline Physical Activity ( Metabolic equivalent) & & $2843.16(3956.39)$ \\
\hline Adherence to plant-based dietary patterns & & $32.56(9.61)$ \\
\hline Sex & & \\
\hline Male & $154(29.1)$ & \\
\hline
\end{tabular}


Female

\section{Educational Level}

No education

Primary

Junior High

Senior High

Training College

Polytechnic

University

Clinical Variables

Systolic blood pressure ( $\mathrm{mmHg}$ )

Diastolic blood pressure $(\mathrm{mmHg})$

$\mathrm{HbA} 1 \mathrm{c} \%$

Fasting Blood Sugar(mmol/L)

Total cholesterol (mmol/L)

Triglycerides $(\mathrm{mmol} / \mathrm{L})$

HDL cholesterol (mmol/L)

LDL cholesterol (mmol/L)

Total Bilirubin $(\mathrm{mg} / \mathrm{dL})$
376(70.9)

202(38.1)

85(16.0)

132(24.9)

67(12.6)

21(4.0)

10(1.9)

13(2.5)
$135.67(20.44)$

$77.79(12.79)$

$8.12(3.34)$

$10.05(4.55)$

$7.18(3.48)$

4.63(14.14)

$1.73(0.90)$

$5.15(3.40)$

$19.69(16.41)$

$N(\%)$ :- Number (percentage), Means (SD):- Mean \pm Standard deviation, BMI: - Body mass index, HbAlc\%:- Glycated hemoglobin, HDL cholesterol: - High density lipoprotein cholesterol, LDL cholesterol: - Low density lipoprotein cholesterol

Table 2: Correlation of demographic characteristics and clinical variable with adherence to plant-based dietary patterns 


\begin{tabular}{|c|c|c|}
\hline Variables & $\begin{array}{l}\text { Adherence to plant-based dietary patterns } \\
\text { (r) }\end{array}$ & p-value \\
\hline $\operatorname{BMI}\left(\mathrm{Kg} / \mathrm{m}^{2}\right)$ & $0.087^{*}$ & 0.045 \\
\hline Systolic blood pressure (mmHg) & -0.025 & 0.568 \\
\hline Diastolic blood pressure (mmHg) & 0.054 & 0.213 \\
\hline Diabetes duration (years) & 0.045 & 0.296 \\
\hline Age (years) & -0.050 & 0.255 \\
\hline Education level & 0.049 & 0.262 \\
\hline Physical Activity & -0.035 & 0.422 \\
\hline Number of cigarette smoke per day & -0.045 & 0.305 \\
\hline $\mathrm{HbA} 1 \mathrm{c} \%$ & $-0.279^{* *}$ & $<0.001$ \\
\hline Fasting blood sugar(mmol/L) & $-0.219 * *$ & $<0.001$ \\
\hline Total cholesterol(mmol/L) & $-0.107^{*}$ & 0.014 \\
\hline Triglycerides (mmol/L) & 0.019 & 0.668 \\
\hline HDL cholesterol(mmol/L) & $-0.091^{*}$ & 0.035 \\
\hline LDL cholesterol(mmol/L) & $-0.105^{*}$ & 0.015 \\
\hline Total Bilirubin & -0.052 & 0.232 \\
\hline
\end{tabular}

Table 3: Association of adherence to moringa, moringa-plantain combined diets with glycemic control

\begin{tabular}{lcccccc}
\hline Variables & $\begin{array}{l}\text { Unstandardized } \\
\text { regression } \\
\text { coefficient( }(\boldsymbol{\beta})\end{array}$ & $\begin{array}{c}\text { Standard error } \\
\text { Plantain Adj. }\end{array}$ & $\begin{array}{l}\text { Standardized } \\
\text { regression } \\
\text { coefficient }(\boldsymbol{\beta})\end{array}$ & $\mathbf{t}$ & $\begin{array}{c}\text { P- } \\
\text { value }\end{array}$ & $\mathbf{9 5 \%}$ CI \\
Yam Adj. & $\mathbf{- 0 . 1 7 1}$ & $\mathbf{0 . 0 7 6}$ & $\mathbf{- 0 . 0 9 8}$ & $\mathbf{- 2 . 2 4 8}$ & $\mathbf{0 . 0 2 5}$ & $\mathbf{- 0 . 3 2 1 , - 0 . 0 2 2}$ \\
Egg Plant Stew Adj. & 0.030 & 0.038 & 0.036 & 0.784 & 0.433 & $-0.045,0.105$ \\
Moringa leaves Adj. & $\mathbf{- 0 . 1 6 8}$ & $\mathbf{0 . 0 8 0}$ & $\mathbf{- 0 . 0 9 5}$ & $\mathbf{- 2 . 0 9 7}$ & $\mathbf{0 . 0 3 6}$ & $\mathbf{- 0 . 3 2 5 , - 0 . 0 1 1}$ \\
Bean Stew Adj. & $\mathbf{- 0 . 2 9 2}$ & $\mathbf{0 . 1 4 5}$ & $\mathbf{- 0 . 1 1 2}$ & $\mathbf{- 2 . 0 1 3}$ & $\mathbf{0 . 0 4 5}$ & $\mathbf{- 0 . 5 7 7 , - 0 . 0 0 7}$ \\
Cucumber Adj. & -0.112 & 0.262 & -0.048 & -0.428 & 0.670 & $-0.633,0.409$ \\
Groundnut Past Adj. & -0.001 & 0.002 & -0.023 & -0.525 & 0.600 & $-0.005,0.003$ \\
Roasted Groundnut Adj. & -0.004 & 0.004 & -0.071 & -1.004 & 0.316 & $-0.012,0.004$ \\
Plantain*Egg Plant diet Adj. & 0.014 & 0.034 & 0.047 & 0.419 & 0.677 & $-0.053,0.081$ \\
Plantain*Moriga diet Adj. & $\mathbf{- 0 . 2 3 1}$ & $\mathbf{0 . 0 9 1}$ & $\mathbf{- 0 . 4 0 6}$ & $\mathbf{- 2 . 5 2 9}$ & $\mathbf{0 . 0 1 3}$ & $\mathbf{- 0 . 4 1 3 , - 0 . 0 4 9}$ \\
Plantain*Beans Stew Adj. & $\mathbf{- 0 . 1 1 3}$ & $\mathbf{0 . 0 3 8}$ & $\mathbf{- 0 . 1 2 8}$ & $\mathbf{- 2 . 9 4 9}$ & $\mathbf{0 . 0 0 3}$ & $\mathbf{- 0 . 1 8 8 , - 0 . 0 3 8}$ \\
Yam*Moriga diet Adj. & -0.134 & 0.129 & -0.045 & -1.039 & 0.299 & $-0.388,0.120$ \\
\hline
\end{tabular}

95\%CI: - 95\% confidence interval; Adj:-Adjusted for the effect of physical activity; Plantain*Moriga diet: Plantain and Moriga diets combination; Plantain*Egg Plant diet: - Plantain and Eggplant diets combination;

Yam*Moriga diet: - Yam and Moringa diet combination; Plantain*Beans: - Plantain and Beans combination

\section{Reference}


1. Association, A.D., Diabetes mellitus: a major risk factor for cardiovascular disease. A joint editorial statement by the American diabetes association; the national heart, lung, and blood institute; the juvenile diabetes foundation international; the national institute of diabetes and digestive and kidney diseases; and the American heart association. Circulation, 1999. 100: p. 1132-1133.

2. Perreault, L., M.K. Boardman, and J. Pak, The association between type 2 diabetes and cardiovascular disease: The "for your sweetheart ${ }^{\text {TM" }}$ survey. Advances in therapy, 2019. 36(3): p. 746-755.

3. Orlich, M.J., et al., Vegetarian dietary patterns and mortality in Adventist Health Study 2. JAMA internal medicine, 2013. 173(13): p. 1230-1238.

4. Orlich, M.J. and G.E. Fraser, Vegetarian diets in the Adventist Health Study 2: a review of initial published findings. The American journal of clinical nutrition, 2014. 100(suppl_1): p. 353S-358S.

5. Fung, T., M. Schulze, and J. Manson, Dietary patterns, meat intake, and the risk of type 2 diabetes in women. ACC Current Journal Review, 2005. 2(14): p. 19.

6. Song, S.J., et al., Dietary patterns based on carbohydrate nutrition are associated with the risk for diabetes and dyslipidemia. Science and Technology, 2012. 2010: p. 0004536.

7. Barnard, N.D., et al., Vegetarian and vegan diets in type 2 diabetes management. Nutrition reviews, 2009. 67(5): p. 255-263.

8. Jenkins, D.J., et al., Type 2 diabetes and the vegetarian diet. The American journal of clinical nutrition, 2003. 78(3): p. 610S-616S.

9. Fraser, G., Vegetarianism and obesity, hypertension, diabetes, and arthritis. Diet, life expectancy, and chronic disease, 2003: p. 129-148.

10. Turner-McGrievy, G. and M. Harris, Key elements of plant-based diets associated with reduced risk of metabolic syndrome. Current diabetes reports, 2014. 14(9): p. 524.

11. Melina, V., W. Craig, and S. Levin, Position of the Academy of Nutrition and Dietetics: vegetarian diets. Journal of the Academy of Nutrition and Dietetics, 2016. 116(12): p. 1970-1980.

12. Craig, W.J., Nutrition concerns and health effects of vegetarian diets. Nutrition in Clinical Practice, 2010. 25(6): p. 613-620.

13. Ferdowsian, H.R. and N.D. Barnard, Effects of plant-based diets on plasma lipids. The American journal of cardiology, 2009. 104(7): p. 947-956.

14. Appleby, P.N., G.K. Davey, and T.J. Key, Hypertension and blood pressure among meat eaters, fish eaters, vegetarians and vegans in EPIC-Oxford. Public health nutrition, 2002. 5(5): p. 645-654.

15. Berkow, S.E. and N. Barnard, Vegetarian diets and weight status. Nutrition reviews, 2006. 64(4): $p$. 175-188.

16. Turner-McGrievy, G.M., N.D. Barnard, and A.R. Scialli, A two-year randomized weight loss trial comparing a vegan diet to a more moderate low-fat diet. Obesity, 2007. 15(9): p. 2276-2281.

17. Knutsen, S.F., Lifestyle and the use of health services. The American journal of clinical nutrition, 1994. 59(5): p. 1171S-1175S.

18. Craig, W.J. and A.R. Mangels, Position of the American Dietetic Association: vegetarian diets. Journal of the American dietetic association, 2009. 109(7): p. 1266.

19. Powers, M.A., et al., Diabetes self-management education and support in type 2 diabetes: a joint position statement of the American Diabetes Association, the American Association of Diabetes Educators, and the Academy of Nutrition and Dietetics. The Diabetes Educator, 2017. 43(1): p. 4053.

20. Amaltinga, A.P.M., Non adherence to diabetic medication among diabetic patients, a case study of Dormaa Hospital Ghana. Science Journal of Public Health, 2017. 5(2): p. 88-97.

21. Farrar, D., et al., Risk factor screening to identify women requiring oral glucose tolerance testing to diagnose gestational diabetes: a systematic review and meta-analysis and analysis of two pregnancy cohorts. PloS one, 2017. 12(4): p. e0175288. 
22. Organization, W.H., Global Physical Activity Questionnaire (GPAQ). Analysis Guide: World Health Organization. 2017.

23. Fleming, J.K., Evaluation of HbA1c on the Roche COBAS Integra 800 closed tube system. Clinical biochemistry, 2007. 40(11): p. 822-827.

24. Addai-Mensah, O., et al., Determination of haematological reference ranges in healthy adults in three regions in Ghana. BioMed research international, 2019. 2019.

25. D'Souza, M.S., et al., From Fad to Fact: Evaluating the Impact of Emerging Diets on the Prevention of Cardiovascular Disease. The American journal of medicine, 2020.

26. Benson, G. and J. Hayes, An update on the Mediterranean, vegetarian, and DASH eating patterns in people with type 2 diabetes. Diabetes Spectrum, 2020. 33(2): p. 125-132.

27. Misra, R., et al., Vegetarian diet and cardiometabolic risk among asian indians in the United States. Journal of diabetes research, 2018. 2018.

28. Petersen, K.S., et al., Healthy dietary patterns for preventing cardiometabolic disease: the role of plant-based foods and animal products. Current developments in nutrition, 2017. 1(12): p. cdn. 117.001289.

29. Qian, F., et al., Association between plant-based dietary patterns and risk of type 2 diabetes: a systematic review and meta-analysis. JAMA internal medicine, 2019. 179(10): p. 1335-1344.

30. Ayodele, O.H. and E.V. Godwin, Glycemic indices of processed unripe plantain (Musa paradisiaca) meals. African Journal of Food Science, 2010. 4(8): p. 514-521.

31. Iweala, E., I. Obichi, and O. Omotosho, Biochemical and histological responses of hepatotoxic rats fed Musa paradisiaca L. supplemented diet. International Journal of Pharmacology, 2011. 4: p. 471477.

32. Flores-Silva, P.C., et al., Gluten-free spaghetti made with chickpea, unripe plantain and maize flours: functional and chemical properties and starch digestibility. International Journal of Food Science \& Technology, 2014. 49(9): p. 1985-1991.

33. Lupsa, B.C. and S.E. Inzucchi, Diabetic ketoacidosis and hyperosmolar hyperglycemic syndrome. Endocrine Emergencies, 2014: p. 15-31.

34. Famakin, O., et al., Assessment of nutritional quality, glycaemic index, antidiabetic and sensory properties of plantain (Musa paradisiaca)-based functional dough meals. Journal of food science and technology, 2016. 53(11): p. 3865-3875.

35. Uhegbu, F., C. Imo, and C. Onwuegbuchulam, Hypoglycemic, Hypolipidemic and Antioxidant Activities of Musa paradisiaca, Normalis (Plantain) Supplemented Diet on Alloxan Induceddiabetic Albino Rats. Asian J Biochem, 2016. 11: p. 162-7.

36. Iroaganachi, M., C. Eleazu, and P. Okafor, Effect of Unripe Plantain (Musa paradisiaca) and Ginger (Zingiber officinale) on Renal Dysfunction in Streptozotocin-Induced Diabetic Rats. JOP. Journal of the Pancreas, 2015. 16(2): p. 167-170.

37. Haber, S.L., et al., Effects of Moringa oleifera in patients with type 2 diabetes. American Journal of Health-System Pharmacy, 2020. 77(22): p. 1834-1837.

38. Hassan, F.A.G. and M.A. Ibrahim, Moringa oleifera: Nature is most nutritious and multi-purpose tree. International Journal of Scientific and Research Publications, 2013. 3(4): p. 1-5.

39. Singh, A.K., et al., Phytochemical, nutraceutical and pharmacological attributes of a functional crop Moringa oleifera Lam: An overview. South African Journal of Botany, 2020. 129: p. 209-220.

40. Nova, E., et al., Potential of Moringa oleifera to Improve Glucose Control for the Prevention of Diabetes and Related Metabolic Alterations: A Systematic Review of Animal and Human Studies. Nutrients, 2020. 12(7): p. 2050. 